\title{
Research on Furnace Temperature Curve Based on Heat Convection and Heat Radiation
}

\author{
Qiaoyun Lei ${ }^{1, *}$, Ziyou Zhang ${ }^{2}$, Songyi Zhuo ${ }^{1}$, and Kangju Lin ${ }^{1}$ \\ ${ }^{1}$ College of Science, Zhejiang University of Technology,310012, China \\ ${ }^{2}$ College of Information Engineering, Zhejiang University of Technology,310012, China
}

\begin{abstract}
Insights into the mechanism of reflow soldering temperature curve, a mathematical model of the temperature change of the circuit board surface is established. The heat transfer methods, heat radiation and heat convection is analyzed in detail. The curve of air temperature inside the reflow furnace is solved by establishing an one-dimensional heat conduction model and fitting coefficients. The heat radiation received by the circuit board mainly comes from the small temperature zone. Depending on the area where the circuit board is located, heat radiation is itemized into 3 types: heat radiation in the gap, the temperature zone and the adjacent temperature zone of the area before and after the furnace. The impacts of heat radiation is weighted and analyzed by relative distance. For coefficients, analyzing and fitting is further discussed.
\end{abstract}

\section{Introduction}

In the processing of electronic products like integrated circuit boards, electronic components are automatically soldered to the circuit board by heating. The reflow soldering machine is widely employed, however, the setting of reflow soldering temperature curve challenges us. At present, plenty works in this area is basically controlled and adjusted through experimental tests. However, this thesis aims to analyze mechanism models.

Inside the reflow furnace, four big temperature zones with varying functions, preheating zone, constant temperature zone, reflow zone, and cooling zone, consist small temperature zones[1]. The circuit board are put on conveyor belt to go through the furnace at a stable speed for heating and welding. By temperature control, the ideal temperature curve is like Figure2[2].

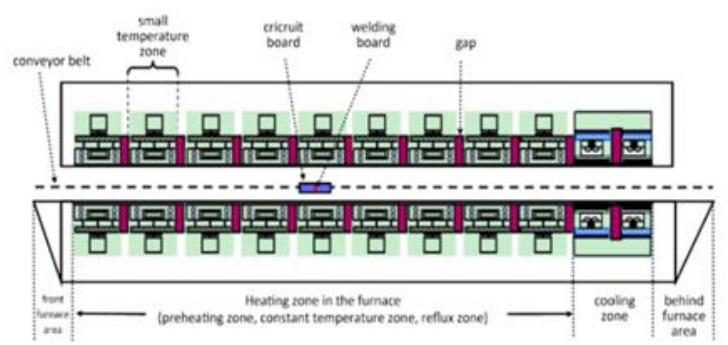

Fig. 1.Schematic diagram of reflow furnace.

\footnotetext{
* Corresponding author: 201806110914@zjut.edu.cn
}

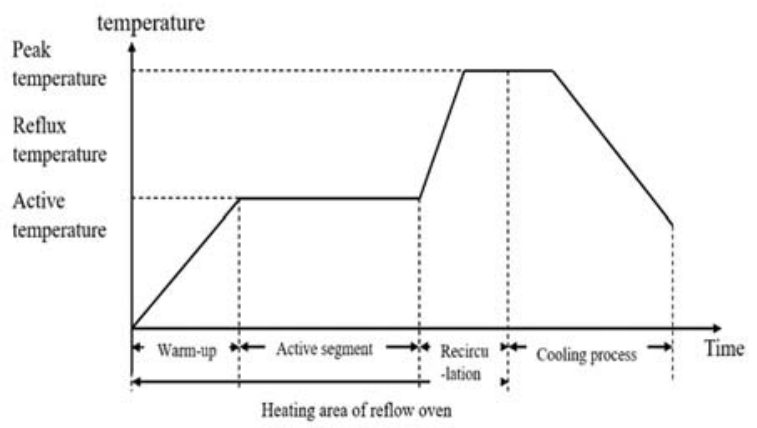

Fig. 2. The ideal temperature curve.

\section{Material and Method}

\subsection{Reflow furnace temperature curve change model}

The temperature change of the welding area involves two aspects: heat conduction and heat convection[3]. The operation of conveyer belt maintains the speed of circuit in the same level. While it passing through five function zones, the heat is being conveyed. First, the stabilization of heating air temperature curve is obtained to be the foundation of following works. Deep insights into the heat conduction and heat convection are explicitly listed below.

\subsubsection{Air temperature curve after stabilization}

For the symmetrical distribution heating devices, we have established a one-dimensional temperature curve. The leftmost end of the furnace front area is set as the origin of the line. 
The gap is heated by the adjacent small temperature zone as no heating device in the gap. The heat conduction of marginal parts is similar to gap. The heat stabilized quickly for conducting through the boundary. A one-dimensional heat conduction model is correspondingly established in Equation(1)[4].

$$
\frac{\partial u_{i j}(x, t)}{\partial t}=k \frac{\partial^{2} u_{i j}(x, t)}{\partial x^{2}}
$$

where $u_{i j}$ is a binary function of coordinate $x$ and time $t$ and $\mathrm{i}, \mathrm{j}$ is ith zone $\mathrm{jth}$ order to respectively label the small temperature zone. $\mathrm{k}$ is the thermal diffusivity, latter discussion is arranged.

$$
\left\{\begin{array}{c}
u_{i j}(0, t)=T_{i} \\
u_{i j}(l, t)=T_{j} \\
u_{i j}(x, 0)=25
\end{array}\right.
$$

where $T_{i}$ is the temperature of right edge in Figure $1, T_{j}$ is left edge temperature, $x$ represents the distance from the left border and $l$ is the length of the conveyer.

As for coefficient $k$, we have $k=\mathrm{K}_{0} / \mathrm{cp}$. In this equation $K_{0}$ is thermal conductivity, $c$ is specific heat capacity and $\rho$ is mass density of the air.

\subsubsection{Heat convection model}

Relative movement is what ought to happen as the circuit is transported and it allows the process of heat convection[5]. At the same time, the air in the furnace itself produces heat convection. However, its influence is so slight that could be ignored. Therefore, this sub-topic does not consider the heat convection generated by the air movement in the furnace.

In most cases, Newton's theorem functions for the basement of the heat transfer of convective motion. The square area of heat transfer is S shown in Equation(3).

$$
q_{c}=h_{c}\left(T_{a}-u_{s}\right)
$$

where $q$ is the transferred heat per unit area, $u$ is the

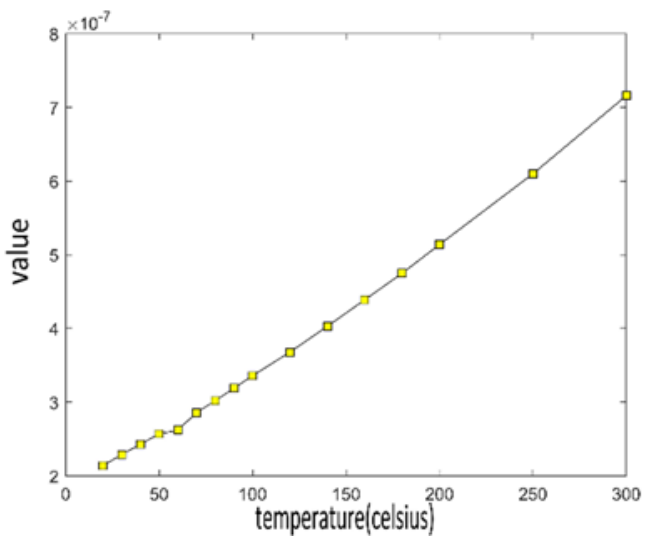

Fig. 3. Thermal conductivity of air

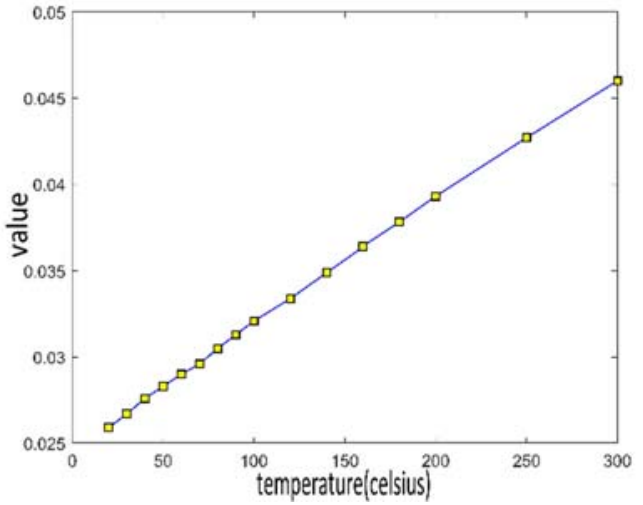

Fig.4. Dynamic viscosity of air;

temperature of the welding surface area and coefficient $h_{c}$ denotes heat convection parameter, we will discuss it later. $T_{a}$ represents the distribution of air temperature in the furnace when it is stable, which has been given in the previous section.

$$
h_{c}=\frac{0.664 \sqrt{v_{f}}\left(c_{p} \eta\right)^{\frac{1}{3}} \lambda^{\frac{2}{3}}}{\sqrt{v L}}
$$

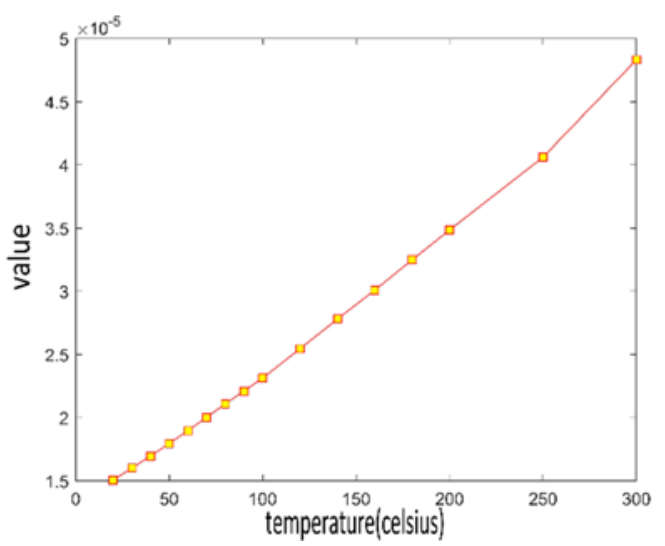

Fig.5. Heat capacity of air.

According to the information reviewed, the above $v, \eta, c_{p}$, and $\lambda$ will change as temperature changes. and

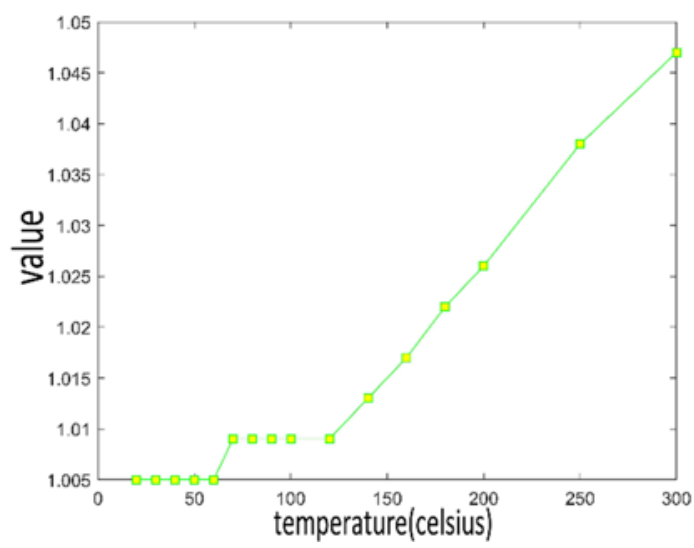

Fig.6. Air fluid of viscosity.

Figure3-6 are the trend respectively of thermal conductivity, dynamic viscosity of, heat capacity and air fluid viscosity.

After obtaining these parameters, the value of the heat 
convection parameter $h_{c}$ at a certain temperature can be determined.

\subsection{Thermal radiation model}

During the operation of the conveyor belt, the heat in the welding area is also affected by the radiation in the small temperature zone. The radiated energy of a small temperature zone is constant. Radiated energy will attenuation as the distance is longer. Assumption that only three nearest small temperature zones has the thermal radiation impact on the welding area is applied.

Case 1 circuit board is in a small temperature zone

When the circuit board is in a certain small temperature zone, it receives thermal radiation energy from the current small temperature zone and the front and rear small temperature zones. Suppose it is in the ith small temperature zone. Then the received radiation energy $q_{r, i}$ is:

$$
\begin{gathered}
q_{r, i}=0.05 h_{r}\left(T_{i-1}^{4}-u_{s}^{4}\right)+0.9 h_{r}\left(T_{i}^{4}-u_{s}^{4}\right)+ \\
0.05 h_{r}\left(T_{i+1}^{4}-u_{s}^{4}\right)
\end{gathered}
$$

Case 2 circuit board located in zone gap

When circuit board being in the gap between the ith and $(i+1)$ th small temperature, its received radiation energy ${ }^{[2]} q_{r, i+0.5}$ is:

$$
q_{r, i+0.5}=0.5 h_{r}\left(T_{i-1}^{4}-u_{s}^{4}\right)+0.5\left(T_{i+1}^{4}-u_{s}^{4}\right)
$$

Case 3 circuit board located in marginal place

The circuit board is regarded as releasing radiant energy into the external environment and cooling down. The radiant energy received at this time $q_{r, 0}$ is:

$$
q_{r, 0}=h_{r}\left(300.15^{4}-u_{s}^{4}\right)
$$

\subsection{Combination of the model}

$$
q=q_{c}+q_{r}
$$

where $q$ is the change in heat per unit area. $q_{c}$ means thermal radiation, $q_{r}$ means thermal convection

The reflow furnace has 11 small temperature zones and 10 gaps. So $q$ can be determined by Equation (9).

$$
\left\{\begin{array}{l}
h_{c}\left(T_{1}-T_{2}\right)+\sum_{i=1}^{3} \omega_{i} h_{r}\left(T_{2}^{4}-u_{s}^{4}\right), x \in p_{i} \\
h_{c}\left(T_{1}-T_{2}\right)+\sum_{i=1}^{2} \omega_{i} h_{r}\left(T_{2}^{4}-u_{s}^{4}\right), x \notin p_{i}
\end{array}\right.
$$

\section{Results}

\subsection{Air temperature Curve in Furnace}

Take step $h_{x}=0.001$ as the discrete step size for the area length, and $h_{t}=0.1$ as the discrete step size for the time. The accepted finite difference method is then used to numerically solve the heat conduction equations (Equation 1).

The air temperature distribution shows a almost linearly upward trend in first small temperature zone. This is more obvious in Figure 8. Even at a time step of $0.1 \mathrm{~s}$, the air temperature will quickly stabilize, and the marginal final temperature will highly approach the temperature of the two temperature zones.

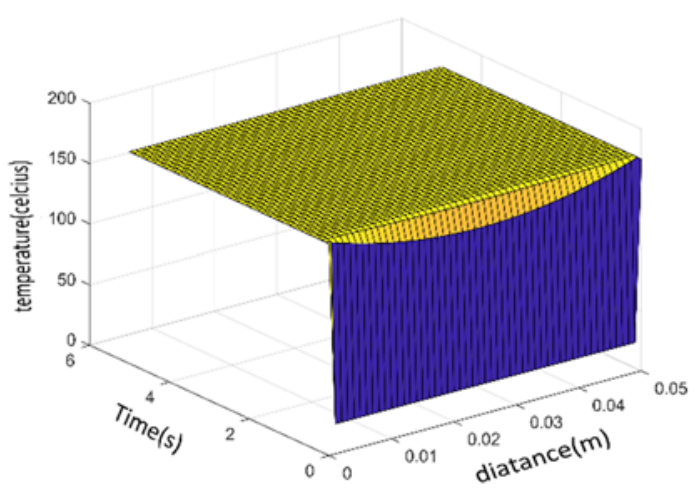

Fig. 7. Air temperature field diagram in front of the furnace

Temperature distribution between gap is basically the same as that in Figure 9 despite their environment. However, the only difference is that as gap range is smaller and the air temperature stabilizes faster. Finally, after solving the air temperature of all gaps and small temperature areas, the air temperature distribution diagram of the reflow furnace can be obtained in Figure 9.

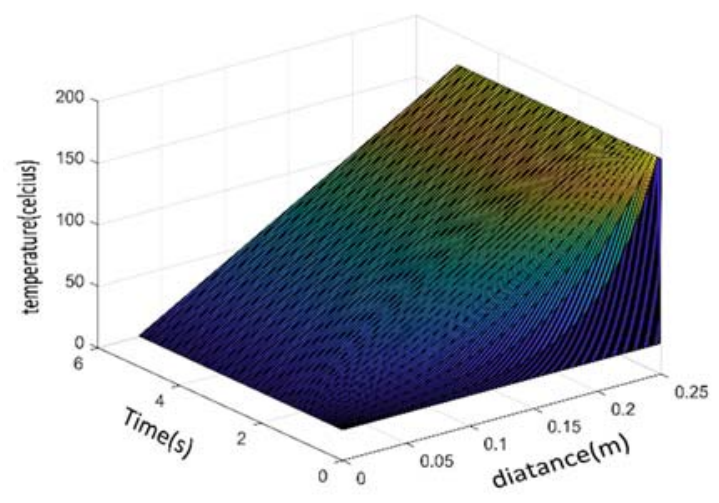

Fig.8.Air temperature field diagram in the end of the furnace

\subsection{Fitting and Determination of Air Parameter}

In the process of real workplace, parameters $c \& m$ (specific heat capacity and mass tensity) can be tested quickly. But on our work, we fitted in segments by numerical integration. 


\subsection{Display of Temperature Curve}

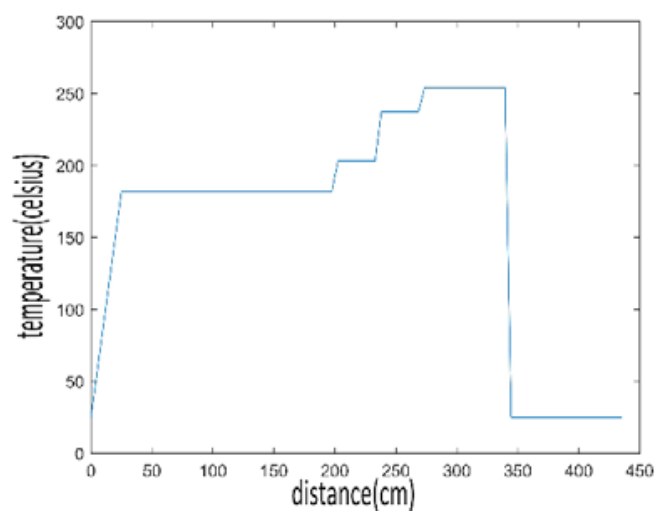

Fig.9. Stable air temperature distribution in reflow furnace

The speed of conveyor belt is set to $78 \mathrm{~cm} / \mathrm{min}$ as we try to figure it out. In Figure10, the temperature curve is visualized in the $\mathrm{x}$-axis of time. The temperature of specific welding rises little in the first twenty seconds and then rises quickly in the heating process. In 110 seconds the temperature is nearly to stay unchanged. Around 200 seconds, the temperature is rising quickly in the process of recirculation. The peak

\section{Conclusion}

Through the thermodynamic research on the furnace temperature curve of the reflow furnace, the mechanism is explored and then obtaining the results of the furnace temperature curve by fitting the analysis parameters of the model. This is instrumental to control the furnace temperature in the actual production process.

\section{References}

1. HP. Wang, The influence of SMT reflow soldering temperature curve on the welding quality [J]. Journal of Qinghai University (Nature Science), 2009, 2.

2. HT. Cai, W. Li, H. Wang, Discussion About the Control of the Temperature Curve of Circumfluence Soldering[J]. Microprocessors, 2008, 29(05): 24-26.

3. BY. Huang, The modeling and simulation of the temperature field of SMT during reflow soldering[D]. Tianjin University, 2005

4. SM. Yang, WQ. Tao,(1982)Heat Transfer [M]. Higher Education Press, Beijing.

5. B. Illés, Measuring heat transfer coefficient in convection reflow ovens[J]. Measurement, 2010, 43(9): 1134-1141. 